\begin{tabular}{|c|c|c|c|c|c|}
\hline MUNIBE Antropologia-Arkeologia & $n^{\circ} 67$ & $269-284$ & DONOSTIA & 2016 & ISSN 1132-2217 • elSSN 2172-4555 \\
\hline
\end{tabular}

\title{
Exploitation du milieu montagnard sur le versant nord des Pyrénées entre 20000 et 12000 cal BP: que nous apprend la faune?
}

\section{Mountain resources exploitation in the northern Pyrenees during the Last Glacial Maximum and the Late Glacial: a focus on faunal remains}

MOTS CLÉS: Magdalénien, Azilien, Tardiglaciaire, Dernier Maximum glaciaire, Archéozoologie.

GAKO-HITZAK: Magdaleniarra, Aziliarra, Izoztaro Berantiarra, Azken Maximo Glaziarra, Arkeozoologia.

KEY WORDS: Magdalenian, Azilian, Late Glacial, Last Glacial Maximum, Zooarchaeology.

\section{Aude CHEVALLIER(1), Sandrine COSTAMAGNO(2), Jean-George FERRIÉ(3), Delphine KUNTZ ${ }^{(4)}$ et Véronique LAROULANDIE ${ }^{(5)}$}

\section{RÉSUMÉ}

Après le LGM, le recul des glaciers pyrénéens a libéré de nouveaux espaces désormais disponibles pour les populations végétales et animales. A travers les données de l'archéozoologie, cet article aborde la fréquentation du versant nord des Pyrénées par les hommes de la fin du Paléolithique. Jusqu'à 15000 cal BP environ, presque toutes les occupations sont situées dans la zone de piémont, en dessous de 500m d'altitude. Des occupations situées entre $500 \mathrm{~m}$ et $1000 \mathrm{~m}$ deviennent ensuite plus fréquentes. Faiblement exploités avant 15000 cal BP, les gibiers de montagne et en particulier le Bouquetin prennent de l'importance à partir du Magdalénien supérieur, ce qui explique sans doute en partie la fréquentation d'altitudes plus élevées. La chasse à la Marmotte bien documentée dans les Alpes, n'a pas d'équivalent dans les Pyrénées. La rareté de ce rongeur dans les Pyrénées pourrait avoir contribué à la faible fréquentation des altitudes élevées.

\section{LABURPENA}

Azken glaziazioaren amaieran, Pirinioetako glaziarrek atzerakada izatean, orduan landare- eta animalia-populazioek hartzen zituzten espazioak okupaziorik gabe gelditu ziren. Artikulu honetan, Pirinioen iparraldeko aldean Paleolitoaren amaierako gizakiak bertan zenbateraino ibiltzen ziren aztertu dugu datu faunistikoen bidez. 15.000 cal BP ingurura arte, aztarnategi gehienak Pirinioen inguruetan daude, $500 \mathrm{~m}$-tik beherako altitudean. Ondoren, ohikoagoak dira 500-1000m-an kokatutako aztarnategiak. 15.000 cal BP baino lehen gutxi aprobetxatzen ziren mendiko harrapakinak, bereziki basahuntza, baina garrantzi handiagoa hartu zuten Goi Magdaleniar garaitik aurrera eta horrek azaltzen du, ziurrenik, altitude garaiagoetako presentzia. Marmotaren ehiza ondo dokumentatuta dago Alpeetan, baina ez du baliokiderik Pirinioetan. Baliteke karraskari hori Pirinioetan bakana izateak zerikusia izatea goi-altitudeetako presentzia txikiarekin.

\section{ABSTRACT}

After the Late Glacial Maximum, the retreat of Pyrenean glacier release new areas for vegetal, animal and human populations. The search of mountain animal resources could have played an important role in the human recapture of high areas at the end of the Paleolithic.

About twenty sites with zooarchaeological data, occupied between c. 21000 and 12000 cal BP and located at an altitude over 200m were analyzed.

Before c. 15000 cal BP (GS-2b and GS-2a), almost all of the occupations are located in the piedmont, under 500m. During the Gl-1 they seem to be deserted for new places higher in the mountain but always under $1000 \mathrm{~m}$. However, a difference seems to appear between the West of the chain, where the glaciers very early disappeared, and the East, where they stayed in low altitude until the Gl-1. In the Arudy basin (Pyrénées-Atlantiques) for example, the same sites, located around $500 \mathrm{~m}$, were occupied during all the period.

No datum of seasonality is available for the oldest phases. During the Middle Magdalenian, while we could expect a frequenting of the Pyrenean sites during the good season, it is on the contrary the bad season which is the best represented. It may suggest that few parameters could have limited the installation of human population at high altitude in summer. From the Gl-1, seasonality data are more various and suggest that the Pyrenees could have been frequented all year round.

During the Badegoulian and the Old Magdalenian, Reindeer is the more frequent game in all the sites. It stays the first prey during the Middle Magdalenian, especially in the East. Horse is also dominant in some sites of the Middle and West Pyrenees. Mountain games are not very exploited. During the Upper Magdalenian, Reindeer is still present in many sites but it's never the first game. Horse or Red deer are sometimes

\footnotetext{
(1) Post-Doctorante. Équipe Ethnologie Préhistorique. UMR 7041 - ArScAn. MAE, 21 allée de I'Université. F-92023 Nanterre Cedex. aude. chevallier@ gmail.com

(2) Directrice de Recherche CNRS. UMR 5608 - TRACES

${ }^{(3)}$ Chercheur indépendant.

(4) Post-doctorante. Équipe Ethnologie Préhistorique. UMR 7041 - ArScAn.

(5) Chargée de Recherche CNRS. UMR-5199 PACEA.
} 
dominant but in the East, it's currently Ibex that was first exploited. During the Azilian, Ibex stay frequent but Red deer is generally dominant. Roe deer and Wild boar are also more frequent. Small preys - leporids like mountain Hare, birds like Ptarmigan or fishes like Salmon - are also exploited, especially from the Upper Magdalenian.

During all the period, no selected mortality profiles are known. The games are generally introduced complete and exploited for meat, marrow but also skin or tendons.

While in the Alps the sites occupied during the GS-2 are rarely located above $300 \mathrm{~m}$, in the Pyrenees we found them frequently between 400 and 500m. During the Gl-1 the sites are higher in the Alps (until 1250m for the Upper Magdalenian and 1500m for the Epipaleolithic) than in the Pyrenees where they never exceed 1000m. Hunting camps specialized on Marmot capture are frequent in the Alps but totally unknown in the Pyrenees. The scarcity of this rodent could explain the low frequentation of Pyrenean summits at the end of the Paleolithic.

\section{1.- INTRODUCTION}

Le recul des glaciers pyrénéens qui s'est amorcé dès le début du stade isotopique 2, a ouvert de nouveaux espaces pour les espèces végétales et animales. Dans cet article, nous nous interrogeons sur le rythme et les motivations de la recolonisation des hauteurs pyrénéennes par les groupes humains à la fin du Paléolithique, en analysant les données de la faune. On peut en effet se demander dans quelle mesure l'exploitation de ressources animales particulières à ce milieu montagnard a pu inciter les hommes à s'aventurer en altitude.

Notre recherche s'est centrée sur le versant nord des Pyrénées pour la période allant de 21000 à 12000 cal BP environ. Cette période est marquée par de nombreux changements climatiques entraînant des conditions plus ou moins favorables à une installation en altitude des hommes et conduisant dans cette région (COSTAMAGNO et al., 2008), comme dans d'autres (DELPECH, 1999; BRIDAULT, et al., 2000; BRUGAL, et al., 2004; SZMIDT, et al., 2009), à une recomposition des biocénoses animales.

Après avoir rappelé brièvement le contexte climatique et environnemental ainsi que les données disponibles sur le désenglacement de la chaîne pyrénéenne, nous présentons la localisation et la chronologie des occupations. Puis, nous focalisons notre attention sur les modalités d'exploitation de la faune en interrogeant différents paramètres: la saisonnalité, les gibiers chassés, le traitement des carcasses et les ressources recherchées. Enfin nous comparons nos résultats avec ce qui est par ailleurs connu à la même époque dans les Alpes en nous interrogeant sur les facteurs responsables des différences observées.

\section{2.- LE CONTEXTE CLIMATIQUE ET ENVIRON- NEMENTAL}

Le GS-2.1b de la chronologie glaciaire débute il y a 21000 ans environ et s'achève à une date dont le calage chronologique reste imprécis (17 500 env., RASMUSSEN, et al., 2014). Dans les Pyrénées, la végétation est caractéristique d'un climat froid et sec. De type steppique, elle est dominée par les herbacés héliophiles comme les Poacées et les Armoises (Artemisia). Durant cette période, les arbres étaient probablement absents des vallées nord-pyrénéennes (JALUT \& TURU I MICHELS, 2009).
Le GS-2.1a qui lui succède (environ $17 \quad 480$-14 $700 \mathrm{~b} 2 \mathrm{k}$ ), est grosso modo contemporain du stade d'Heinrich 1 (HS1, selon les limites de HARRISON \& SANCHEZ GOÑI, 2010) enregistré dans les carottes marines et qui se traduit notamment par une débâcle glaciaire et un refroidissement des eaux de surface. Sur le continent, le Dryas ancien correspond également à cette période (NAUGHTON, et al., 2009). La végétation, caractérisée par une augmentation de la proportion des Armoises et par le développement du Genévrier (Juniperus), traduit une amélioration des conditions climatiques (WALKER, 1995). L'ambiance générale reste toutefois encore assez froide, ne permettant pas le développement d'un véritable couvert forestier (JALUT \& TURU I MICHELS, op. cit.).

Avec le réchauffement du Gl-1 (interstade Bølling-Allerød, environ 14480 - 12896 b2k), une période de forte instabilité climatique commence (RASMUSSEN, et al., 2006; RASMUSSEN, et al., 2014). Au cours du Bølling, avec une augmentation rapide des températures, certains arbres se développent rapidement comme le Genévrier et le Bouleau (Betula) alors que les Armoises diminuent. Le milieu reste encore largement ouvert (JALUT \& TURU I MICHELS, op. cit.). Malgré un refroidissement enregistré tant au niveau global que local, le couvert forestier devient plus dense à l'Allerød, avec en particulier le fort développement du Pin (Pinus) et, dans une moindre mesure, du Bouleau. Le Chêne (Quercus) fait son apparition (ibid.).

La péjoration climatique du GS-1 (Dryas récent, environ 12896 - 11703 b2k, RASMUSSEN et al., op. cit.) est marquée quant à elle par une recrudescence des herbacées héliophiles et un recul des arbres (JALUT \& TURU I MICHELS, op. cit.).

Des variations locales sont toutefois à noter tout au long de la période, avec un climat plus sec et froid dans la partie occidentale de la chaîne et plus doux et humide à l'Est (DELMAS, et al., 2011)

\section{3.- METODOLOGÍA}

Ce climat très contrasté a fortement influencé l'emprise des glaces dans les différentes vallées pyrénéennes et le rythme de leur désenglacement. Ainsi, à l'ouest de la chaîne, dans les vallées dugave de Pau, du gave d'Ossau ou le bassin de la Garonne, on ob- 
serve un retrait rapide des glaces entre 30 à 26000 BP. Durant le LGM, les glaciers ne réinvestissent pas les basses vallées: les bassins d'Arudy ou de Lourdes restent libres de glace. Au contraire, dans la partie est - notamment dans le Bassin de l'Ariège - l'extension du glacier est très importante au LGM. Au Dryas ancien, le bassin de Tarascon est encore totalement englacé et le front de glace s'étend jusqu'à Ax-les-Thermes, à 700 m d'altitude. Le retrait des glaces, amorcé dans cette partie de la chaîne à partir de la fin du GS-2, est en revanche très rapide: au Bølling, les glaciers sont présents uniquement dans les cirques et leur fonte est complète à l'Allerød (DELMAS et al., op. cit.).

\section{4.- CORPUS}

Une vingtaine de sites ont été sélectionnés en fonction de différents critères: seuls les gisements implantés à plus de 200 m d'altitude, avec une attribution chrono-culturelle bien établie, bien datés par le radiocarbone et pour lesquels nous disposons de données archéozoologiques quantitatives ont été retenus (Figure 1 et Tableau 1).

Longtemps, les phases les plus anciennes - le Badegoulien et le Magdalénien inférieur - sont restées quasiment inconnues dans les Pyrénées françaises. Depuis quelques années, les révisions entreprises sur un certain nombre de sites, comme par exemple ce- lui des Scilles (PÉTILLON, et al., 2008; LANGLAIS, et al., 2010), tendent à pallier cette lacune. Outre celui-ci, attribué au Magdalénien inférieur et situé en Haute-Garonne, seule la couche 4 d'Enlène en Ariège, attribuée au Badegoulien (FOSSE, 1992), a livré à ce jour suffisamment de données pour être intégrée au corpus.

Les phases plus récentes - Magdalénien moyen et supérieur - sont mieux documentées, avec des occupations tout le long de la chaîne (tableau 1).

En raison des critères retenus, l'Azilien est représenté dans cette étude par deux sites seulement: Troubat et Rhodes II. Ce groupe culturel est attesté sur d'autres sites du versant nord de la chaîne, comme le Mas d'Azil (PIETTE, 1895) ou la Tourasse (ORLIAC, 1975), toutefois les données concernant la faune y sont encore trop limitées pour autoriser une incorporation au corpus.

\section{5.- LE RYTHME DE RECOLONISATION DES HAUTEURS PYRÉNÉENNES: LOCALISATION ET DATATION DES OCCUPATIONS DU LGM ET DU TARDIGLACIAIRE}

En représentant graphiquement les données du radiocarbone (Figure 2), les différentes phases culturelles s'ordonnent globalement assez bien dans le temps: le Badegoulien puis le Magdalénien inférieur se placent au GS-2b, le Magdalénien moyen pendant le GS2-a, le Magdalénien supérieur débute légèrement avant le Bø-

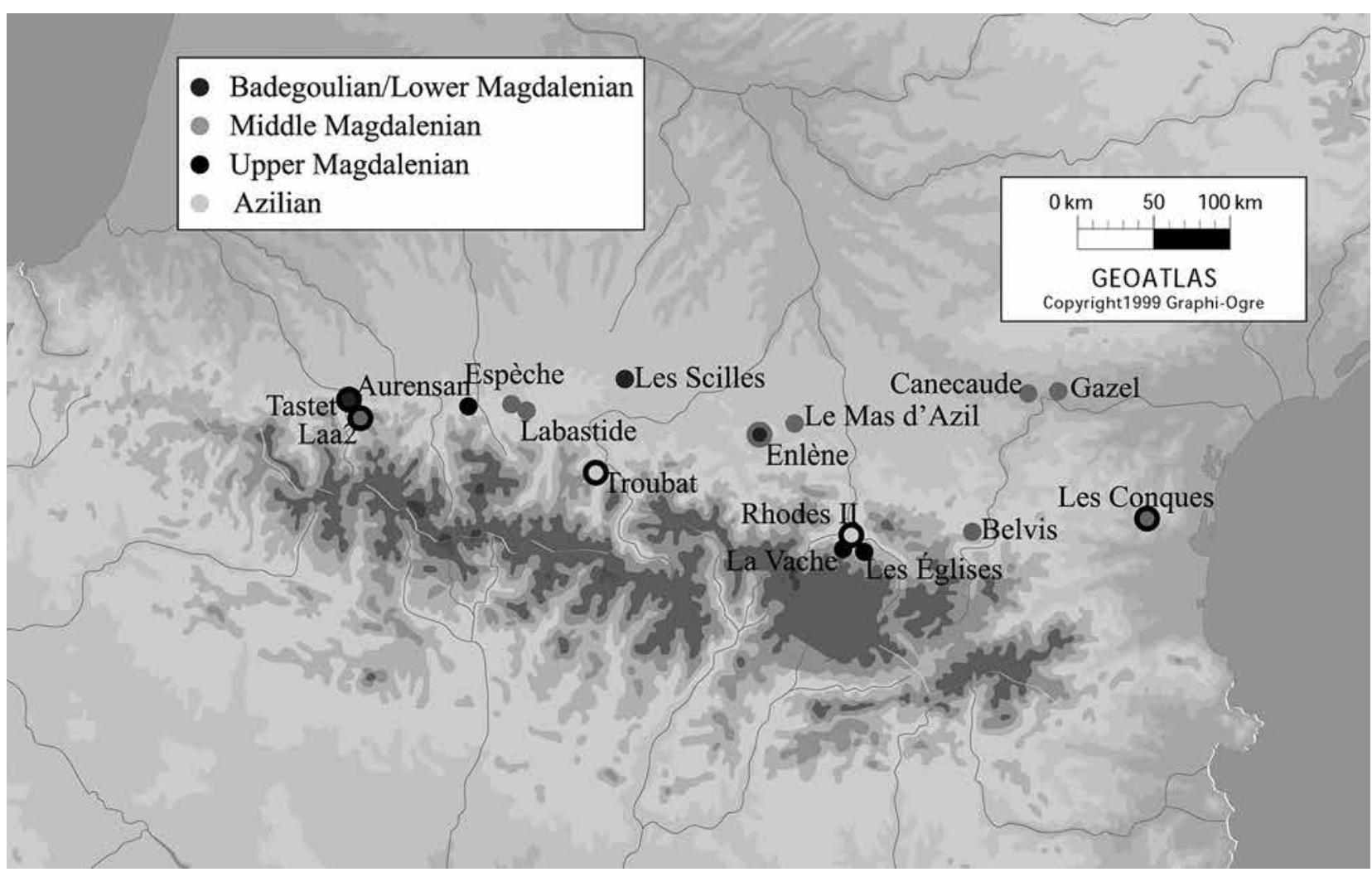

Fig. 1. Carte des sites utilisés dans cette étude / Map of the sites analysed in this study. 


\begin{tabular}{|c|c|c|c|c|c|c|c|}
\hline Site & Commune & Département & Altitude & Niveau & Attribution & Datations & Source \\
\hline \multirow{4}{*}{ Laa2 } & \multirow{4}{*}{ Arudy } & \multirow{4}{*}{ Pyrénées-Atlantiques } & \multirow{4}{*}{$457 \mathrm{~m}$} & 2 & $\begin{array}{l}\text { Magdalénien } \\
\text { supérieur }\end{array}$ & $\begin{array}{c}13370 \pm 70 \mathrm{BP} \\
\text { (Poz-52969); } \\
13550 \pm 60 \mathrm{BP} \\
\text { (OXA-26674); } \\
12552 \pm 83 \mathrm{BP} \\
\text { (Erl-1112) }\end{array}$ & \multirow{4}{*}{$\begin{array}{l}\text { PÉTILLON et } \\
\text { al., } 2015\end{array}$} \\
\hline & & & & 3 & $\begin{array}{l}\text { Magdalénien } \\
\text { moyen }\end{array}$ & $\begin{array}{c}13665 \pm 60 \mathrm{BP} \\
\text { (OxA-26673); } \\
14252 \pm 94 \mathrm{BP} \\
\text { (Erl-11113); } \\
14570 \pm 65 \mathrm{BP} \\
\text { (OxA-26672) }\end{array}$ & \\
\hline & & & & 4 & $\begin{array}{l}\text { Magdalénien } \\
\text { moyen? }\end{array}$ & & \\
\hline & & & & 5 & $?$ & $\begin{array}{c}16380 \pm 80 \mathrm{BP} \\
\text { (OxA-27395); } \\
16070 \pm 75 \mathrm{BP} \\
(\mathrm{O} \times \mathrm{A}-26671) ; \\
16555 \pm 75 \mathrm{BP} \\
(\mathrm{O} \times \mathrm{A}-29934)\end{array}$ & \\
\hline \multirow{3}{*}{ Tastet } & \multirow{3}{*}{ Sainte-Colome } & \multirow{3}{*}{ Pyrénées-Atlantiques } & \multirow{3}{*}{$512 \mathrm{~m}$} & 205 & $\begin{array}{l}\text { Magdalénien } \\
\text { supérieur }\end{array}$ & $\begin{array}{c}14270 \pm 80 \mathrm{BP} \\
(\mathrm{Ly}-10027)\end{array}$ & \multirow{3}{*}{$\begin{array}{l}\text { PÉTILLON et al., } \\
2015\end{array}$} \\
\hline & & & & 207 & $\begin{array}{l}\text { Magdalénien } \\
\text { inférieur/moyen }\end{array}$ & $\begin{array}{c}15800 \pm 90 \mathrm{BP} \\
(\text { Ly- 10028) }\end{array}$ & \\
\hline & & & & 305 & $\begin{array}{l}\text { Magdalénien } \\
\text { moyen }\end{array}$ & $\begin{array}{c}13930 \pm 70 \mathrm{BP} \\
(\text { Ly- 10029) }\end{array}$ & \\
\hline Aurensan & $\begin{array}{l}\text { Bagnères-de-Bi- } \\
\text { gorre }\end{array}$ & Hautes-Pyrénées & $570 m$ & & $\begin{array}{l}\text { Magdalénien } \\
\text { supérieur }\end{array}$ & $\begin{array}{l}13910 \pm 230 \\
\operatorname{BP}(L Y-1107)\end{array}$ & $\begin{array}{c}\text { CLOT \& OMNÈS, } \\
\text { 1979; CLOT, } \\
1983\end{array}$ \\
\hline Bois de Cantet & Espèche & Hautes-Pyrénées & $380 \mathrm{~m}$ & & $\begin{array}{l}\text { Magdalénien } \\
\text { moyen }\end{array}$ & $\begin{array}{l}13060 \pm 430 \\
\text { BP }(\text { Ly-1404) }\end{array}$ & CLOT, 1984 \\
\hline Labastide & Labastide & Hautes-Pyrénées & 550 m & & $\begin{array}{l}\text { Magdalénien } \\
\text { moyen }\end{array}$ & $\begin{array}{l}14260 \pm 440 \\
\text { BP (Ly-1405) }\end{array}$ & CLOT, 1982 \\
\hline \multirow{4}{*}{ Troubat } & \multirow{4}{*}{ Troubat } & \multirow{4}{*}{ Hautes-Pyrénées } & \multirow{4}{*}{$541 \mathrm{~m}$} & 11 à 13 & $\begin{array}{l}\text { Magdalénien } \\
\text { moyen }\end{array}$ & & $\begin{array}{c}\text { COSTAMAGNO, } \\
\text { inédit }\end{array}$ \\
\hline & & & & 8 & $\begin{array}{l}\text { Magdalénien } \\
\text { supérieur }\end{array}$ & $\begin{array}{c}12860 \pm 60 \mathrm{BP} \\
\text { (Lyon-9969/ } \\
\text { SacA32594); } \\
13040 \pm 60 \mathrm{BP} \\
\text { (Lyon-9970/ } \\
\text { SacA32595) }\end{array}$ & $\begin{array}{l}\text { CHEVALLIER, } \\
\text { thèse en cours }\end{array}$ \\
\hline & & & & 7 & $\begin{array}{l}\text { Magdalénien } \\
\text { terminal }\end{array}$ & $\begin{array}{l}11520 \pm 100 \\
\text { BP (OxA-913) }\end{array}$ & $\begin{array}{c}\text { COSTAMAGNO } \\
2005\end{array}$ \\
\hline & & & & 6 & Azilien récent & $\begin{array}{c}10225 \pm 45 \mathrm{BP} \\
\text { (Lyon-9968/ } \\
\text { SACA32593); } \\
10770 \pm 100 \\
\text { BP (Ly-5275) }\end{array}$ & $\begin{array}{l}\text { FERRIÉ, thèse } \\
\text { en cours }\end{array}$ \\
\hline Les Scilles & Lespugue & Haute-Garonne & $330 \mathrm{~m}$ & & $\begin{array}{l}\text { Magdalénien } \\
\text { inférieur }\end{array}$ & $\begin{array}{c}16180 \pm 140 \\
\text { BP (TO-13555) }\end{array}$ & $\begin{array}{l}\text { PÉTILLON et al., } \\
\text { 2008; LANGLAIS } \\
\text { et al., } 2010\end{array}$ \\
\hline \multirow[t]{2}{*}{ Enlène } & $\begin{array}{c}\text { Montes- } \\
\text { quieu-Avantès }\end{array}$ & Ariège & $550 \mathrm{~m}$ & 3 ET 2 & $\begin{array}{l}\text { MAGDALÉNIEN } \\
\text { MOYEN }\end{array}$ & $\begin{array}{l}13940 \pm 250 \\
\text { BP (Gif- } 4124) \\
13900 \pm 120 \\
\text { BP (Gif- } 6030) \\
13400 \pm 120 \\
\text { BP (Gif- } 5770) \\
12900 \pm 140 \\
\text { BP (Gif-5321) }\end{array}$ & FOSSE, 1992 \\
\hline & & & & 4 & Badegoulien & & \\
\hline Les Églises & Ussat & Ariège & $628 \mathrm{~m}$ & & $\begin{array}{l}\text { Magdalénien } \\
\text { supérieur }\end{array}$ & $\begin{array}{l}12900 \pm 220 \\
\text { BP (Gif-3923) } \\
11800 \pm 500 \\
\text { BP (Gif-1434) }\end{array}$ & $\begin{array}{l}\text { DELPECH \& LE } \\
\text { GALL, } 1983\end{array}$ \\
\hline Le Mas d'Azil & Le Mas d'Azil & Ariège & $410 \mathrm{~m}$ & rive Droite & $\begin{array}{l}\text { Magdalénien } \\
\text { moyen }\end{array}$ & $\begin{array}{l}13640 \pm 110 \\
\text { BP (Gif-5522) } \\
13200 \pm 110 \\
\text { BP (Gif-5680) }\end{array}$ & PATOU, 1984 \\
\hline
\end{tabular}




\begin{tabular}{|c|c|c|c|c|c|c|c|}
\hline La Vache & Alliat & Ariège & $578 \mathrm{~m}$ & $\begin{array}{l}\text { salle } \\
\text { Monique }\end{array}$ & $\begin{array}{l}\text { Magdalénien } \\
\text { supérieur }\end{array}$ & $\begin{array}{c}13650 \pm 130 \\
\text { BP (GifA-96480) } \\
13490 \pm 120 \\
\mathrm{BP}(\mathrm{GifA}-96478) \\
12850 \pm 30 \\
\mathrm{BP}(\mathrm{GrN}-2026) \\
12540 \pm 105 \\
\mathrm{BP}(\mathrm{GrN}-2025) \\
11650 \pm \\
200(\mathrm{~L}-336 \mathrm{c}) \\
12800 \pm 140 \\
\mathrm{BP}(\mathrm{Gif}-7603)\end{array}$ & $\begin{array}{l}\text { PAILHAUGUE, } \\
1998 ; 2004\end{array}$ \\
\hline \multirow{4}{*}{ Rhodes II } & \multirow{4}{*}{ Arignac } & \multirow{4}{*}{ Ariège } & \multirow{4}{*}{$520 \mathrm{~m}$} & F1 à 4 & $\begin{array}{l}\text { Magdalénien } \\
\text { supérieur }\end{array}$ & $\begin{array}{c}\text { F1: } 12530 \pm \\
\text { 50 (Lyon-9964 } \\
\text { (SacA32589); } \\
\text { F3: } 12450 \pm \\
\text { 60 (Lyon-9965 } \\
\text { (SacA32590) }\end{array}$ & $\begin{array}{c}\text { DELPECH, 1983; } \\
\text { BOUCHARD, } \\
2008\end{array}$ \\
\hline & & & & F5 & Azilien ancien & $\begin{array}{l}11770 \pm 50 \\
\text { (Lyon-9967 } \\
\text { (SacA32592) }\end{array}$ & $\begin{array}{l}\text { CHEVALLIER, } \\
\text { thèse en cours }\end{array}$ \\
\hline & & & & F6 & Azilien récent & $\begin{array}{l}11310 \pm 50 \\
\text { (Lyon-9966 } \\
\text { (SacA32591) }\end{array}$ & $\begin{array}{l}\text { CHEVALLIER, } \\
\text { thèse en cours }\end{array}$ \\
\hline & & & & F7 & Azilien récent & $\begin{array}{c}10630 \pm 45 \\
(\text { Lyon-10255 } \\
\text { (SacA 33730) }\end{array}$ & $\begin{array}{l}\text { CHEVALLIER, } \\
\text { thèse en cours }\end{array}$ \\
\hline Canecaude & Villardonnel & Aude & $250 \mathrm{~m}$ & 2 & $\begin{array}{l}\text { Magdalénien } \\
\text { moyen }\end{array}$ & $\begin{array}{c}13935 \pm 45 \mathrm{BP} \\
(\mathrm{KIA}-25340)\end{array}$ & FONTANA, 1999 \\
\hline Belvis & Belvis & Aude & $960 \mathrm{~m}$ & 3 & $\begin{array}{l}\text { Magdalénien } \\
\text { supérieur }\end{array}$ & $\begin{array}{c}13080 \pm 50 \mathrm{BP} \\
\text { (SacA-6973) } \\
12330 \pm 50 \mathrm{BP} \\
\text { (SacA-6971) } \\
12270 \pm 280 \mathrm{BP} \\
\text { (Gif-2950) }\end{array}$ & FONTANA, 1999 \\
\hline Gazel & $\begin{array}{l}\text { Sallèles- } \\
\text { Cabardès }\end{array}$ & Aude & $200 \mathrm{~m}$ & & $\begin{array}{l}\text { Magdalénien } \\
\text { moyen }\end{array}$ & $\begin{array}{c}14462 \pm 62 \mathrm{BP} \\
(\mathrm{KIA}-22856)\end{array}$ & FONTANA, 1999 \\
\hline Les Conques & Vingrau & Pyrénées-Orientales & $200 \mathrm{M}$ & & $\begin{array}{l}\text { Magdalénien } \\
\text { moyen }\end{array}$ & $\begin{array}{c}14320 \pm 90 \mathrm{BP} \\
(\text { Ly-8779) }\end{array}$ & MOIGNE, 2003 \\
\hline
\end{tabular}

Tableau 1: Liste des sites utilisés dans cette étude/ List of the sites analysed in this study

lling (début du Gl-1) et l'Azilien se développe à l'Allerød et au Dryas récent (fin du Gl-1 et GS-1).

Quelques anomalies méritent toutefois d'être discutées. L'occupation d'Aurensan est datée du GS-2a, pourtant l'étude de l'industrie osseuse tendrait plutôt à la rattacher au Magdalénien supérieur (BAUMANN, 2006). Le Mas d'Azil, attribué au Magdalénien moyen, a quant à lui fourni des âges comparables à ceux des occupations du Magdalénien supérieur. Pour la Vache, attribuée au Magdalénien supérieur, les datations couvrent une période assez vaste, entre 16923 et 13100 cal BP. Pour ces gisements fouillés anciennement, on peut s'interroger sur la fiabilité des datations, souvent réalisées selon la méthode conventionnelle qui conduit à des écarts-types importants ainsi que sur l'homogénéité culturelle des assemblages.

La couche 7 de Troubat, attribuée à un Magdalénien terminal, peut être rattachée à l'Allerød d'après les datations radiocarbone, ce qui a conduit M. Barbaza (BARBAZA, 1996; 2011) à émettre I'hypothèse d'une possible contemporanéité entre les dernières manifestations magdaléniennes et les premiers témoignages aziliens.
Avant 15000 cal BP (GS-2), au Badegoulien et au Magdalénien inférieur et moyen, la plupart des occupations est localisée à moins de 500m d'altitude et ce sont essentiellement des sites de piémont qui semblent avoir été fréquentés. La grotte de Tastet (512m d'altitude) dans les Pyrénées-Atlantiques - occupée dès le Magdalénien inférieur - ainsi que celles de Troubat (541m) et Labastide (550m) dans les Hautes-Pyrénées et Enlène (550m) en Ariège, fréquentées à partir du Magdalénien moyen, font toutefois figure d'exception. Après $15000 \mathrm{cal} \mathrm{BP} \mathrm{(Gl-1} \mathrm{et}$ GS-1), au contraire, seules des occupations situées audelà des $500 \mathrm{~m}$ d'altitude sont représentées dans notre corpus, exception faite de Laa2 (457m) dans le Bassin d'Arudy et des Conques (200m) dans l'Aude. Si la majorité des occupations du Magdalénien supérieur et de l'Azilien est située entre 500 et $600 \mathrm{~m}$ d'altitude, la grotte des Églises est localisée à $628 \mathrm{~m}$ et celle de Belvis à 960m d'altitude. Aucun gisement situé à plus de $1000 \mathrm{~m}$ d'altitude n'est cependant connu.

À l'ouest de la chaîne, dans le Bassin d'Arudy, une certaine continuité de peuplement est perceptible tout au long de la période considérée, puisque des gisements occupés dès le GS-2, comme Tastet ou Laa2, le sont également au GI-1 (PÉTILLON et al., 2015). Le retrait 


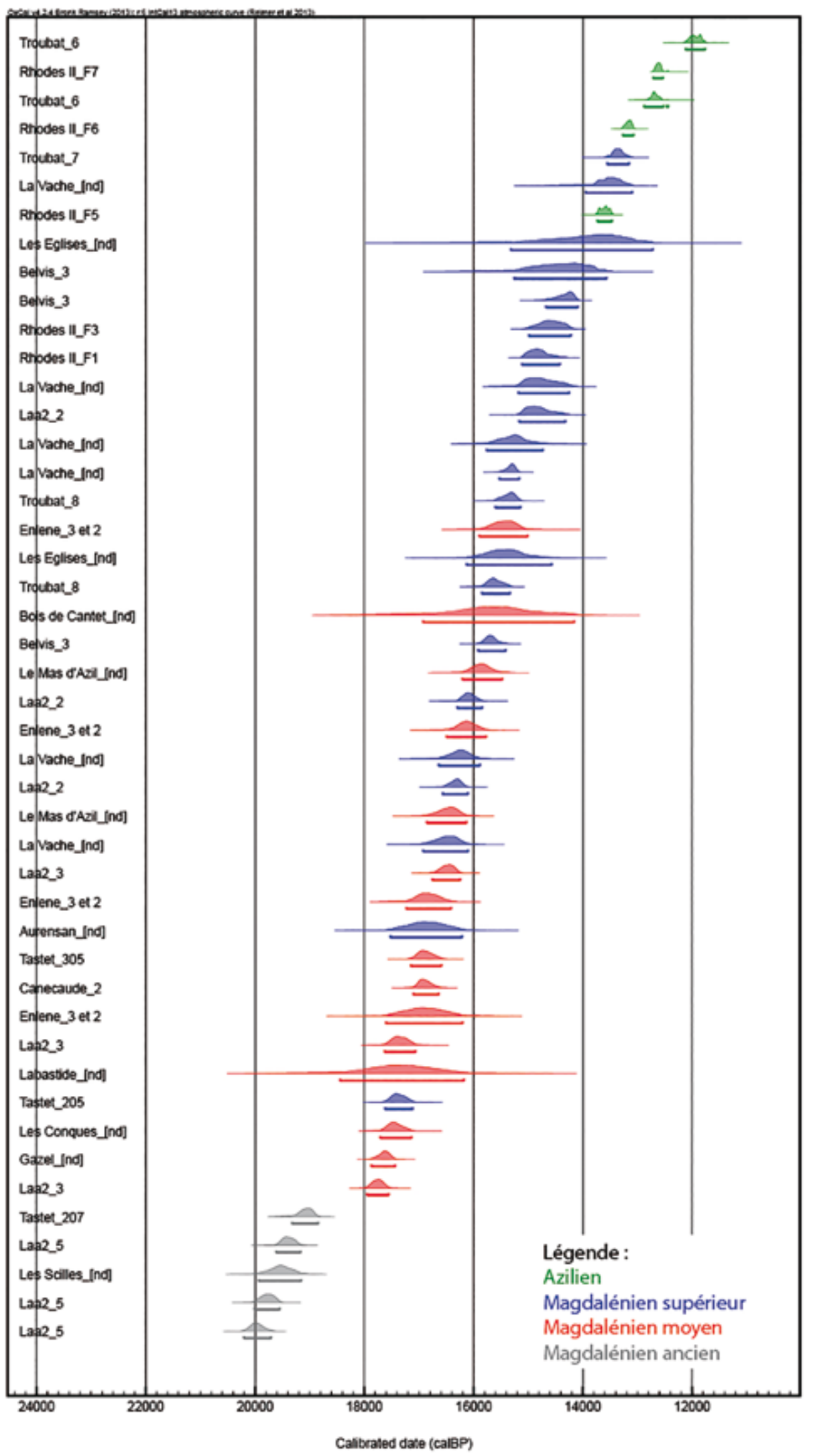

Fig. 2. Les datations radiocarbone (pour le détail des dates, voir tableau 1) / Radiocarbon data (show Table 1 for details about the datations). 
précoce des glaciers dans cette région n'y est sans doute pas étranger. Dans les Hautes-Pyrénées, la grotte-abri de Troubat, brièvement fréquentée au Magdalénien moyen, n'est largement investie qu'à partir du Gl-1 (Magdalénien supérieur). Dans la partie orientale de la chaîne, en revanche, la plupart des sites occupés au GS-2 ne semblent plus l'être au Gl-1 et ce sont alors de nouvelles stations, localisées plus en altitude, qui sont investies. La grotte des Conques (Pyrénées orientales), implantée à 200m d'altitude, est toutefois un contre-exemple puisqu'elle a livré aussi bien du Magdalénien moyen que supérieur.

Si les choix qui ont présidé à l'élaboration de notre corpus conduisent sans doute à une image un peu caricaturale de la situation, elle suggère une évolution des conditions locales de peuplement qu'il est tentant de mettre en lien avec la présence tardive des glaciers dans ces vallées et leur retrait rapide à partir du Gl-1.

\section{LA SAISONNALITÉ DES OCCUPATIONS}

Pour le Badegoulien et le Magdalénien inférieur, aucune donnée de saisonnalité n'est disponible.

Au Magdalénien moyen, certains sites semblent être occupés pendant une large partie de l'année comme Canecaude, (FONTANA, 1999), Gazel (ibid.; MARTIN \& MARTIN, 2010) ou le Mas d'Azil (PATOU, 1984). Dans ces différents gisements, les épisodes maximum de chasses paraissent toutefois s'être déroulés pendant la mauvaise saison (Tableau 2), ce qui est également le cas à Laa2 (PÉTILLON et al., 2015). Seul le site des Conques témoigne d'une occupation limitée à la fin de la belle saison et pourrait correspondre à une halte de chasse (MOIGNE, 2003).

Au cours du Bølling, au Magdalénien supérieur, les indices de saisonnalité sont plus variés. Les chasses se sont préférentiellement déroulées en automne aux

\begin{tabular}{|c|c|c|c|c|c|c|c|c|c|c|c|c|c|c|c|c|c|}
\hline Période & Département & Site & Couche & \multicolumn{12}{|c|}{ indices de saisonnalité } & & \\
\hline & \multirow{3}{*}{ Pyrénées-Atlantiques } & \multirow{6}{*}{ Laa2 } & \multirow{3}{*}{4} & & $J$ & $\mathrm{~F}$ & M & A & $\mathrm{M}$ & & & & A & S & O & N & $\mathrm{D}$ \\
\hline & & & & fœtus (Cheval) & & & & & & & & & & & & & \\
\hline & & & & Renne & & & & & & & & & & & & & \\
\hline & & & \multirow{3}{*}{3} & & J & $\mathrm{F}$ & M & A & M & & & & $A$ & $S$ & 0 & $\mathrm{~N}$ & $\mathrm{D}$ \\
\hline & & & & fœetus (Cerf/Renne) & & & & & & & & & & & & & \\
\hline & & & & fœtus (Bison) & & & & & & & & & & & & & \\
\hline & \multirow{3}{*}{ Ariège } & \multirow{3}{*}{ Mas d'Azil } & \multirow{3}{*}{ Rive droite } & & $\mathrm{J}$ & $\mathrm{F}$ & M & A & M & & & J & A & S & 0 & $\mathrm{~N}$ & $\mathrm{D}$ \\
\hline & & & & Renne & & & & & & & & & & & & & \\
\hline & & & & Cerf & & & & & & & & & & & & & \\
\hline & & \multirow{5}{*}{ Canecaude } & & & J & $\mathrm{F}$ & M & A & M & & & & A & S & 0 & N & $\mathrm{D}$ \\
\hline & & & & Renne & & & & & & & & & & & & & \\
\hline & & & & Isard & & & & & & & & & & & & & \\
\hline & & & & Cheval & & & & & & & & & & & & & \\
\hline \multirow{16}{*}{$\begin{array}{l}\text { Magdalénien } \\
\text { moyen }\end{array}$} & & & & Poissons & & & & & & & & & & & & & \\
\hline & \multirow{15}{*}{ Aude } & \multirow{11}{*}{ Gazel } & \multirow{11}{*}{ 7-7bis } & & J & $\mathrm{F}$ & M & A & M & J & & J & A & S & $\mathrm{O}$ & N & D \\
\hline & & & & Renne & & & & & & & & & & & & & \\
\hline & & & & Cheval & & & & & & & & & & & & & \\
\hline & & & & Bouquetin & & & & & & & & & & & & & \\
\hline & & & & Bovinés & & & & & & & & & & & & & \\
\hline & & & & Poissons & & & & & & & & & & & & & \\
\hline & & & & Renne (cémento) & & & & & & & & & & & & & \\
\hline & & & & Bovinés (cémento) & & & & & & & & & & & & & \\
\hline & & & & Bouquetin (cémento) & & & & & & & & & & & & & \\
\hline & & & & Cheval (cémento) & & & & & & & & & & & & & \\
\hline & & & & Isard (cémento) & & & & & & & & & & & & & \\
\hline & & & & & J & $\mathrm{F}$ & M & A & M & J & te & & A & S & O & N & $\mathrm{D}$ \\
\hline & & Jes ronguus & 3 & Renne & & & & & & & & & & & & & \\
\hline & & & & Bouquetin & & & & & & & & & & & & & \\
\hline & & & & Cerf & & & & & & & & & & & & & \\
\hline
\end{tabular}




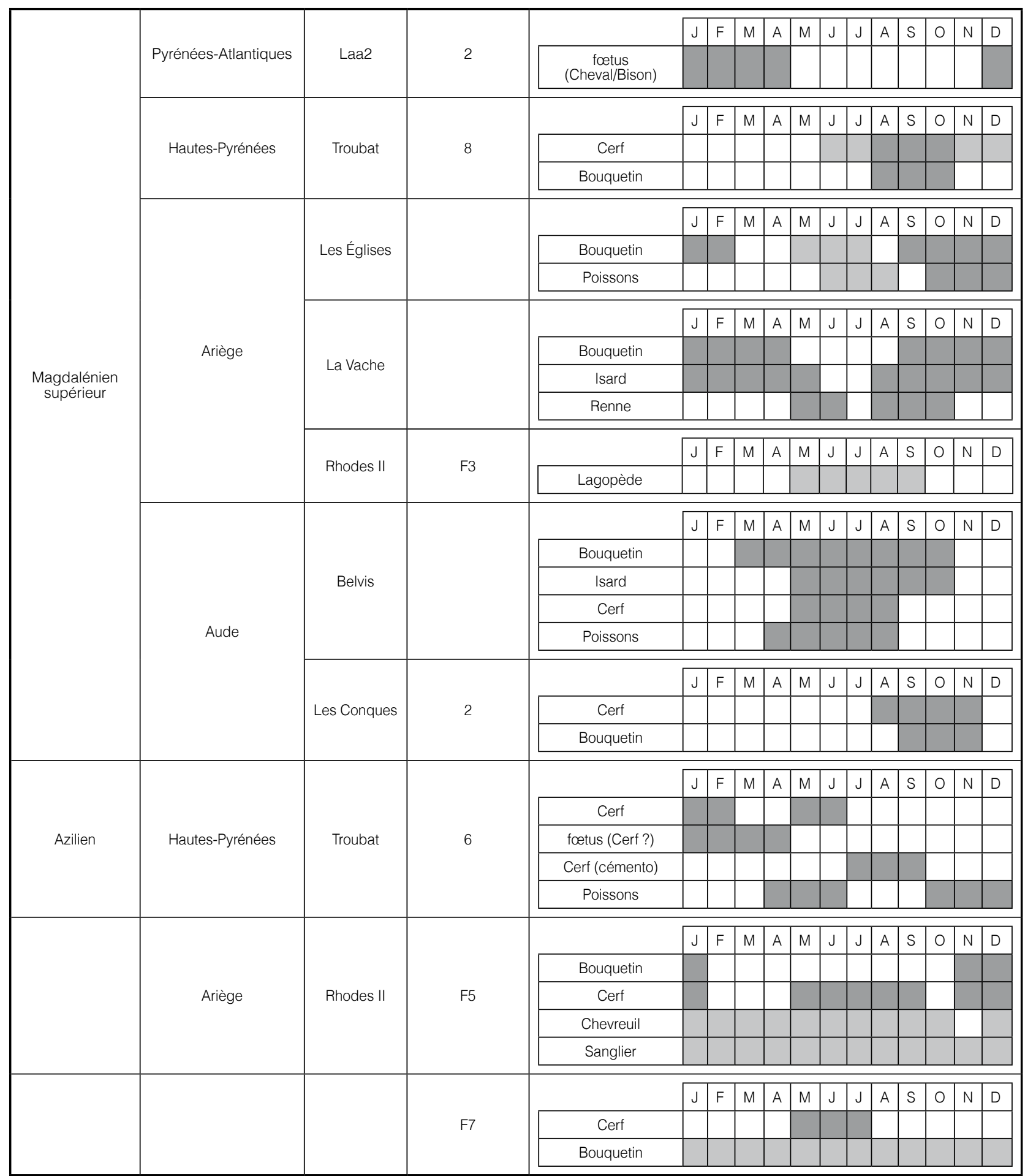

Églises (DELPECH \& LE GALL, 1983) et à Troubat c. 8 (CHEVALLIER, thèse en cours), plutôt en hiver à Laa $2 \mathrm{c}$. 2 (PÉTILLON et al. 2015) et à La Vache (PAILHAUGUE, 1996; 2004). Une chasse estivale aux Lagopèdes est par ailleurs vraisemblable pour le niveau F3 de Rhodes II (BOUCHARD, 2008); cette hypothèse reposant sur la présence d'un seul os immature, n'exclut toutefois pas des épisodes de chasse à d'autres saisons.

À l'Allerød, les indices de saisonnalité obtenus pour le niveau F5 (Azilien ancien) de Rhodes II couvrent une grande partie de l'année, tandis que le niveau F7 (Azilien récent) du même site présente au GS-1 des indices da- 
vantage limités à la bonne saison (CHEVALLIER, thèse en cours). Pour l'Azilien récent de la couche 6 de Troubat, les différents indices de saisonnalité témoignent de chasses aux Ongulés durant la bonne et la mauvaise saison (MARTIN, 1994; FERRIÉ, thèse en cours), et une acquisition de poissons au début et à la fin de la bonne saison (LE GALL, 1999).

Pendant les phases d'occupation les plus anciennes, alors que l'on aurait pu s'attendre à une fréquentation des sites pyrénéens au cours de la bonne saison, c'est au contraire la mauvaise saison qui est la mieux représentée. Cela signifie-t-il que différents paramètres limitaient l'installation des hommes en altitude au cours de la bonne saison (fonte des glaces, marécages, ...)? De courtes incursions estivales plus près des sommets, qui auraient laissé peu de traces, sont toutefois envisageables, comme le suggère la fréquentation très brève du gisement de Troubat à $541 \mathrm{~m}$ d'altitude au Magdalénien moyen (COSTAMAGNO, inédit). À partir du Gl-1, les indices de saisonnalité apparaissent à la fois plus resserrés à l'échelle du site et plus variés à l'échelle régionale, ce qui pourrait indiquer une fréquentation plus permanente des Pyrénées, notamment au-dessus de 500m d'altitude, mais aussi, peut-être, une mobilité accrue. Toutefois, le site de Belvis, localisé à 960m d'altitude, semble plutôt correspondre à un site résidentiel, contrairement à celui des Eglises, à 628m d'altitude, qui pourrait s'apparenter à une halte de chasse.

\section{LES GIBIERS CHASSÉS}

Au cours du Pléniglaciaire, le Renne (Rangifer tarandus) est le gibier dominant sur tous les sites pour lesquels des données sont disponibles, tant en NR qu'en NMI (Tableau 3). Aux Scilles (PÉTILLON, et al., 2008; LANGLAIS, et al., 2010) et à Enlène (FOSSE, 1992), il est accompagné par les Bovinés (Bos ou Bison) et l'Isard (Rupicapra pyrenaica).

\begin{tabular}{|c|c|c|c|c|c|c|c|c|c|c|c|c|c|c|}
\hline Période & Département & Site & Couche & & 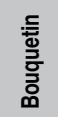 & $\begin{array}{l}\text { 롫 } \\
\text { 요 }\end{array}$ & $\begin{array}{l}\overline{\widetilde{J}} \\
\text { 造 }\end{array}$ & $\stackrel{\mathscr{\Xi}}{\stackrel{\mathscr{\Xi}}{\partial}}$ & 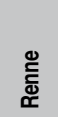 & ङั & 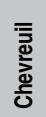 & 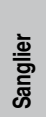 & 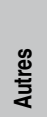 & 咞 \\
\hline \multirow{2}{*}{ Badegoulien } & \multirow{2}{*}{ Ariège } & \multirow{2}{*}{ Enlène } & \multirow{2}{*}{4} & NR & & 4 & 15 & 68 & 264 & & & & & 351 \\
\hline & & & & $\% N R$ & & 1,1 & 4,3 & 19,4 & 75,2 & & & & & \\
\hline \multirow{4}{*}{ Magdalénien inférieur } & \multirow{2}{*}{ Haute-Garonne } & \multirow{2}{*}{ Les Scilles } & & NR & & 14 & 9 & 21 & 40 & 3 & & & 2 & 89 \\
\hline & & & & $\% N R$ & & 16 & 10 & 24 & 45 & 3 & & & 2 & \\
\hline & \multirow{2}{*}{ Pyrénées-Atlantiques } & \multirow{2}{*}{ Tastet } & \multirow{2}{*}{207} & $\mathrm{NR}$ & & & & & 1 & 3 & & & & 4 \\
\hline & & & & $\% N R$ & & & & & 25 & 75 & & & & \\
\hline \multirow{22}{*}{ Magdalénien moyen } & \multirow{6}{*}{ Pyrénées-Atlantiques } & \multirow{2}{*}{ Tastet } & \multirow{2}{*}{305} & NR & & & 14 & 2 & 20 & & & & & 36 \\
\hline & & & & $\% N R$ & & & 38,9 & 5,6 & 55,6 & & & & & \\
\hline & & \multirow{4}{*}{ Laa2 } & \multirow{2}{*}{4} & NR & 16 & 26 & 7 & 5 & 1 & 3 & & & & 58 \\
\hline & & & & $\% N R$ & 27,6 & 44,8 & 12,1 & 8,6 & 1,7 & 5,2 & & & & \\
\hline & & & \multirow{2}{*}{3} & NR & 76 & 34 & 173 & 8 & 117 & 23 & & & & 431 \\
\hline & & & & $\% \mathrm{NR}$ & 17,6 & 7,9 & 40,1 & 1,9 & 27,1 & 5,3 & & & & \\
\hline & \multirow{8}{*}{ Hautes-Pyrénées } & \multirow{2}{*}{ Aurensan } & & NR & 33 & 50 & 33 & 13 & 11 & 16 & 3 & & & 159 \\
\hline & & & & $\% N R$ & 20,8 & 31,4 & 20,8 & 8,2 & 6,9 & 10,1 & 1,9 & & & \\
\hline & & \multirow{2}{*}{ Bois de Cantet } & & NR & 5 & 37 & 2 & 18 & 156 & 36 & 2 & & 8 & 264 \\
\hline & & & & $\% N R$ & 1,9 & 14,0 & 0,8 & 6,8 & 59,1 & 13,6 & 0,8 & & 3,0 & \\
\hline & & \multirow{2}{*}{ Labastide } & & NR & 8 & 4 & 60 & 56 & 28 & & & & & 156 \\
\hline & & & & $\% N R$ & 5,1 & 2,6 & 38,5 & 35,9 & 17,9 & & & & & \\
\hline & & \multirow{2}{*}{ Troubat } & \multirow{2}{*}{11 à 13} & $\mathrm{NR}$ & 18 & & 5 & 12 & 8 & & & & & 43 \\
\hline & & & & $\% \mathrm{NR}$ & 41,9 & & 11,6 & 27,9 & 18,6 & & & & & \\
\hline & \multirow{2}{*}{ Ariège } & \multirow{2}{*}{ Mas d'Azil } & \multirow{2}{*}{ Rive droite } & $\mathrm{NR}$ & 20 & 13 & 174 & 176 & 1268 & 464 & 2 & 2 & & 2119 \\
\hline & & & & $\% \mathrm{NR}$ & 0,9 & 0,6 & 8,2 & 8,3 & 59,8 & 21,9 & 0,1 & 0,1 & & \\
\hline & \multirow{6}{*}{ Aude } & Canocaude & & NR & 57 & 72 & 131 & 148 & 5878 & 13 & & & & 6299 \\
\hline & & Laliectande & & $\% \mathrm{NR}$ & 0,9 & 1,1 & 2,1 & 2,3 & 93,3 & 0,2 & & & & \\
\hline & & Corat & $77 \mathrm{hir}$ - & NR & 157 & 17 & 1366 & 94 & 6647 & 20 & & & & 8301 \\
\hline & & galzel & $1-1 \mathrm{DIS}$ & $\% \mathrm{NR}$ & 1,9 & 0,2 & 16,5 & 1,1 & 80,1 & 0,2 & & & & \\
\hline & & top Congun & 0 & NR & & & & & $s$ de do & & & & & \\
\hline & & Les conques & 3 & $\% \mathrm{NR}$ & 23 & 1 & 3 & 2 & 45 & 25 & 0 & 1 & 1 & \\
\hline
\end{tabular}




\begin{tabular}{|c|c|c|c|c|c|c|c|c|c|c|c|c|c|c|}
\hline \multirow{12}{*}{ Magdalénien supérieur } & \multirow{2}{*}{ Pyrénées-Atlantiques } & \multirow{2}{*}{ Laa2 } & \multirow{2}{*}{2} & NR & 24 & 9 & 193 & 1 & 81 & 39 & 2 & & & 349 \\
\hline & & & & $\% N R$ & 6,9 & 2,6 & 55,3 & 0,3 & 23,2 & 11,2 & 0,6 & & & \\
\hline & \multirow{2}{*}{ Hautes-Pyrénées } & \multirow{2}{*}{ Troubat } & \multirow{2}{*}{8} & NR & 516 & 186 & 10 & 14 & 39 & 626 & 61 & 3 & & 1455 \\
\hline & & & & $\% N R$ & 35,5 & 12,8 & 0,7 & 1,0 & 2,7 & 43,0 & 4,2 & 0,2 & & \\
\hline & \multirow{4}{*}{ Ariège } & \multirow{2}{*}{ Les Églises } & & NR & 9085 & 1 & & & 7 & 2 & 2 & & & 9097 \\
\hline & & & & $\% \mathrm{NR}$ & 99,9 & 0,0 & & & 0,1 & 0,0 & 0,0 & & & \\
\hline & & \multirow{2}{*}{ La Vache } & \multirow{2}{*}{ salle Monique } & NR & 71451 & 3307 & 16 & 206 & 5821 & 755 & 14 & 33 & & 81603 \\
\hline & & & & $\% N R$ & 87,6 & 4,1 & 0,0 & 0,3 & 7,1 & 0,9 & 0,0 & 0,0 & & \\
\hline & \multirow{4}{*}{ Aude } & \multirow{2}{*}{ Belvis } & & NR & 1512 & 158 & 205 & 23 & 2 & 213 & & & & 2113 \\
\hline & & & & $\% N R$ & 71,6 & 7,5 & 9,7 & 1,1 & 0,1 & 10,1 & & & & \\
\hline & & Les Con- & ? & NR & \multicolumn{10}{|c|}{ pas de données } \\
\hline & & ques & 2 & $\% \mathrm{NR}$ & 27 & 0 & 6 & 1 & 46 & 17 & 0 & 2 & 0 & \\
\hline \multirow{2}{*}{ Magdalénien terminal } & \multirow{2}{*}{ Hautes-Pyrénées } & \multirow{2}{*}{ Troubat } & \multirow{2}{*}{$7 a$} & NR & 262 & 183 & 8 & 7 & & 296 & 179 & 77 & & 1012 \\
\hline & & & & $\% N R$ & 25,9 & 18,1 & 0,8 & 0,7 & & 29,2 & 17,7 & 7,6 & & \\
\hline \multirow{8}{*}{ Azilien } & \multirow{2}{*}{ Hautes-Pyrénées } & \multirow{2}{*}{ Troubat } & \multirow{2}{*}{6} & NR & 3113 & 1279 & 2 & 99 & 2 & 4580 & 864 & 1209 & & 11148 \\
\hline & & & & $\% \mathrm{NR}$ & 27,9 & 11,5 & 0,0 & 0,9 & 0,0 & 41,1 & 7,8 & 10,8 & & \\
\hline & \multirow{6}{*}{ Ariège } & \multirow{6}{*}{ Rhodes II } & \multirow{2}{*}{ F5 } & NR & 228 & 17 & & 2 & & 134 & 56 & 30 & & 467 \\
\hline & & & & $\% N R$ & 48,8 & 3,6 & & 0,4 & & 28,7 & 12,0 & 6,4 & & \\
\hline & & & \multirow{2}{*}{ F6 } & NR & 27 & 4 & & & & 92 & 15 & 28 & & 166 \\
\hline & & & & $\% \mathrm{NR}$ & 16,3 & 2,4 & & & & 55,4 & 9,0 & 16,9 & & \\
\hline & & & \multirow{2}{*}{ F7 } & NR & 48 & & & & & 278 & 79 & 107 & & 512 \\
\hline & & & & $\% N R$ & 9,4 & & & & & 54,3 & 15,4 & 20,9 & & \\
\hline
\end{tabular}

Tableau 3: Spectres de faune / Faunal spectra..

Le Renne reste le premier Ongulé exploité au Magdalénien moyen, notamment à l'est de la chaîne comme à Canecaude et Gazel (FONTANA, 1999). Le Cheval (Equus caballus) domine toutefois dans certains sites des Pyrénées-Atlantiques comme Laa 2 (PÉTILLON, et al., 2015) et des Hautes-Pyrénées comme Labastide (CLOT, 1982). Les Caprinés sont en général peu exploités au Magdalénien moyen, malgré quelques exceptions. Deux assemblages sont ainsi dominés par l'Isard: Aurensan (CLOT \& OMNĖS, 1979) et la couche 4 de Laa 2 (PÉTILLON et al., 2015). Pour cette dernière, le faible nombre de restes déterminés invite toutefois à la prudence. Le gisement d'Aurensan, quant à lui, n'est pas sans poser de problèmes, concernant d'une part son attribution chrono-culturelle, comme nous l'avons déjà évoqué, et d'autre part quant à l'origine des restes d'Isard car de nombreux ossements de Carnivores sont présents (Clot, 1983). Le Bouquetin domine quant à lui dans les couches 13 à 11 de Troubat, malgré un nombre de restes réduit qui suggèrerait une fréquentation très éphémère au Magdalénien moyen (COSTAMAGNO, inédit). Discret dans la plupart des autres assemblages, le Bouquetin est toutefois bien représenté dans les niveaux dominés par l'Isard, ainsi qu'aux Conques à l'est de la chaîne (MOIGNE, 2003). On note également la présence significative du Cerf (Cervus elaphus) à Espèche (CLOT, 1984) et au Mas d'Azil (PATOU, 1984), mais il s'agit dans les deux cas de fouilles anciennes.
Au cours du Bølling, le Renne ne domine plus sur aucun des sites considérés même si sa présence est toujours signalée dans presque tous les assemblages. À l'ouest de la chaîne, le Cheval est prépondérant dans la couche 2 de Laa 2 (PÉTILLON et al., 2015). Dans la couche 8 de Troubat, le Cerf est le premier gibier chassé, suivi de près par le Bouquetin (CHEVALLIER, thèse en cours). S'agissant de la période pendant laquelle les cortèges fauniques sont en pleine recomposition, il est possible que de légères différences chronologiques expliquent ces variations. À l'est de la chaîne, en Ariège et dans l'Aude, c'est le Bouquetin qui domine, souvent très largement comme à la Vache (PAILHAUGUE, 1996; 2004), aux Églises (DELPECH \& LE GALL, 1983) ou à Belvis (FONTANA, 1999).

À l'Allerød et au Dryas récent, le Cerf domine à nouveau dans les couches 7 (Magdalénien terminal) et 6 (Azilien récent) de Troubat où il est toujours talonné par le Bouquetin (COSTAMAGNO, 2005; FERRIÉ, thèse en cours). Le Bouquetin domine dans l'Azilien ancien du niveau F5 de Rhodes II, suivi de près par le Cerf, qui le devance dans les niveaux F6 et F7 à l'Azilien récent (CHEVALLIER, thèse en cours). L'Isard est également bien représenté, notamment à Troubat (COSTAMAGNO, 2005; FERRIÉ, thèse en cours). Par rapport aux périodes précédentes, on note la proportion croissante du Chevreuil (Capreolus capreolus) et du Sanglier (Sus scrofa). 
En résumé, au Badegoulien et au Magdalénien inférieur, les gibiers de montagne semblent assez peu chassés. Bouquetins et isards sont mieux documentés au Magdalénien moyen mais leur exploitation semble encore limitée. Au Magdalénien supérieur, le Bouquetin paraît avoir été une ressource de choix, notamment à l'est de la chaîne. Bien qu'encore largement chassé, il est progressivement supplanté par le Cerf à l'Azilien. Néanmoins, non loin de la zone retenue dans cette étude, sur le versant sud des Pyrénées, le site de la Balma Margineda, à 970m d'altitude, apparaît comme une halte saisonnière de chasse au Bouquetin tout au long de I'Azilien (GARDEISEN, 2007; MARTIN, 2007).

La place des petits gibiers est quant à elle très variable selon les gisements. C'est surtout à partir du Magdalénien supérieur que ces proies prennent de l'importance. En effet au Magdalénien moyen, seule la grotte de Gazel témoigne d'une exploitation importante du Lièvre variable (Lepus timidus) pour sa peau mais aussi sa viande (FONTANA, 2003). Ce site livre également plusieurs centaines d'os d'oiseaux qui appartiennent pour la majorité d'entre eux aux Lagopèdes (Lagopus sp.) suivis du Chocard (Phyrrhocorax graculus) et montrent des traces de consommation (VILETTE, 1999; LAROULANDIE, 2009). À Aurensan, le Renard (Vulpes vulpes) est l'espèce la plus abondante (Clot 1983) mais l'absence d'étude taphonomique interroge quant à son origine. Au Magdalénien supérieur en revanche, de nombreux gisements témoignent d'une exploitation accrue de la petite faune (COSTAMAGNO \& LAROULANDIE, 2004; COSTAMAGNO, et al., 2008). Ces sites sont plutôt localisés dans les franges centre et est de la chaîne. Parmi les oiseaux, le Chocard a été chassé sur de nombreux sites mais c'est le Lagopède, en particulier dans la vallée de l'Ariège, qui semble avoir été la cible privilégiée (LAROULANDIE, 2003). Aux Églises (LAROULANDIE, 1998), à la Vache (LAROULANDIE, 2000; 2005) et à Rhodes II (BOUCHARD, 2008), de nombreux individus attestent de l'importance de cette espèce au Magdalénien supérieur. La pêche est également une activité qui se développe surtout à partir du Magdalénien supérieur (LE GALL, 1999; 2003). À Troubat (LE GALL, 1999) ou aux Églises (DELPECH \& LE GALL, 1983), on trouve de nombreux indices de pêche au saumon sur les frayères à la fin de la bonne saison. Le même constat est fait pour l'Azilien de Troubat (LE GALL, 1999) ou de la Balma Margineda (LE GALL, 2007).

\section{LES MODALITÉS D'EXPLOITATION DES GI- BIERS ET LES PRODUITS RECHERCHÉS}

À toutes les périodes, les profils de mortalité indiquent fréquemment une absence de sélection en termes d'âge. On note toutefois une préférence pour les juvéniles et les adultes dans la force de l'âge dans le Magdalénien moyen de Laa 2 (KUNTZ, inédit) ou des Conques (MOIGNE, 2003), dans le Magdalénien supérieur des Églises (DELPECH \& LE GALL, 1983) ou dans l'Azilien de Troubat (FERRIÉ, thèse en cours).
Les carcasses ont souvent été amenées relativement complètes sur les sites, mais il existe quelques assemblages (par exemple Laa2, KUNTZ, inédit) pour lesquels les parties les plus riches en moelle semblent avoir été préférentiellement introduites.

En général les carcasses d'Ongulés ont fait l'objet d'une exploitation intensive conduisant au prélèvement de la peau, à une décarnisation souvent importante, à une récupération systématique de la moelle des os longs, mais aussi fréquemment de celle de la mandibule et des phalanges (Tableau 4). Le prélèvement des tendons est moins systématiquement documenté.

Aucune évolution diachronique ne semble à ce jour perceptible concernant les modalités d'exploitation des gibiers ou les ressources recherchées.

\section{COMPARAISON AVEC LES ALPES}

Dans les Alpes, au GS-2, les occupations humaines sont connues uniquement en dessous de $300 \mathrm{~m}$ - à l'exception du gisement de La Fru situé à 570m d'altitude (MEVEL, 2010) - alors que dans les Pyrénées les gisements sont fréquemment situés entre 400 et $500 \mathrm{~m}$. Comme dans les Pyrénées, le Renne et le Cheval dominent les spectres de faune (BRIDAULT \& CHAIX, 2009). Viennent ensuite, dans les Alpes, le Bouquetin et la Marmotte (Marmota marmota, ibid.). Cette dernière semble en revanche quasiment absente des spectres pyrénéens. Cinq restes sont néanmoins signalés dans le Magdalénien supérieur de La Vache (PAILHAUGUE, 1996). Le Chamois (Rupicapra rupicapra) est par ailleurs très rare dans les archéofaunes des Alpes alors que l'Isard est bien attesté dans les Pyrénées.

Au Gl-1, le nombre de sites connus dans les Alpes augmente considérablement. Ils s'étagent à des altitudes plus élevées que dans les Pyrénées, jusqu'à 1250m au col de la Charmette au Magdalénien et $1500 \mathrm{~m}$ au Clos-de-Lans à l'Épipaléolithique (MONIN, et al., 2006). Si le Bouquetin est fréquemment chassé dans les Alpes, il constitue rarement la proie privilégiée des chasseurs (BRIDAULT \& CHAIX, 2009), contrairement aux Pyrénées. Deux exceptions doivent toutefois être signalées dans les Alpes françaises: les grottes du Taï (SOAVE, 1998) et de Jean-Pierre 1 (LEQUATRE, 1994). En Italie, à Villabruna, les Ongulés de montagne sont les proies les plus fréquemment chassées mais c'est le Chamois et non le Bouquetin qui prédomine (PHOCA-COSMETATOU, 2005). Dans les niveaux épigravettiens de Dalmeri (Allerød), le Bouquetin domine toutefois à plus de 90\% (PHOCA-COSMETATOU, 2009). Cette brève occupation, fréquentée à la fin de la bonne saison, n'est pas sans rappeler la grotte des Églises.

De nombreux sites des Alpes, notamment dans le Vercors, correspondent à des haltes de chasse spécialisées dans l'exploitation de la Marmotte (TOMÉ \& CHAIX, 2003; TOMÉ, 2005; MONIN, et al., 2006), totalement inconnues sur le versant nord des Pyrénées. 


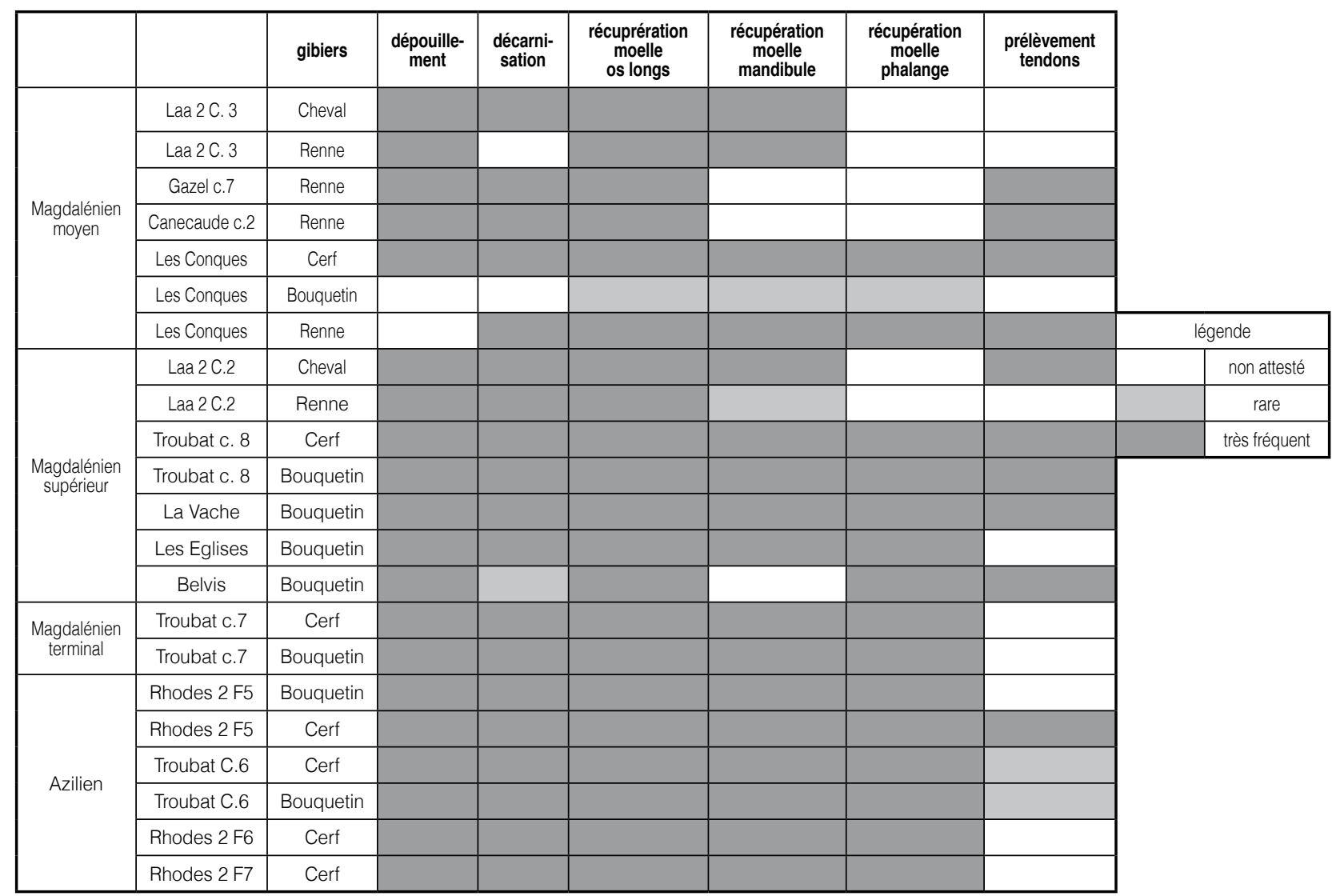

Tableau 4: Les activités documentées / Documented activities.

S'étageant entre 500 et 1050m d'altitude, la plupart de ces sites, comme la grotte Colomb (TOMÉ, 2005), sont situés dans l'étage subalpin de l'époque, au dessus de la limite supérieure de la forêt. On peut s'interroger sur l'absence de sites à marmottes dans les Pyrénées, alors que cette espèce y était présente au Pléistocène au moins jusqu'à environ 14000 cal BP (CLOT \& DURANTHON, 1990). Dans notre corpus, seul le site de Belvis dépasse les 900m d'altitude, aussi peut-on se demander dans quelle mesure l'absence de sites spécialisés sur la Marmotte ne pourrait pas être liée à un problème de conservation et/ou d'identification des sites de hauteur. Cependant, dans les Alpes, des restes de Marmotte sont fréquemment trouvés dans les sites de piémont, ce qui n'est presque jamais le cas dans les Pyrénées. Les effectifs des populations de marmottes étaient-ils déjà trop critiques à la fin du Pléistocène pour faire de cette espèce une ressource intéressante ? Et, de fait, la rareté de sites de haute altitude ne pourrait-elle pas être liée à l'absence ou la rareté de cette espèce dans les Pyrénées ?

Une autre différence par rapport aux Alpes concerne le statut des occupations. Dans le Massif alpin, les sites de moyenne et « haute » montagne correspondent fréquemment à des haltes saisonnières de courtes durées, en particulier les sites à marmottes et bouquetins. Dans les Pyrénées, ce type de sites est peu fréquent:
Les Églises, la Balma Margineda et peut-être Les Conques sont toutefois de bons candidats pour le Gl-1. Au GS-2a, Troubat témoigne aussi d'une courte incursion des Magdaléniens moyens dans le massif montagneux mais, dès le Magdalénien supérieur, la densité des occupations, les structures foyères et les activités documentées par les industries lithiques et osseuses indiquent des occupations, certes saisonnières, mais beaucoup plus longues. La plupart des sites du corpus font donc figure de camps résidentiels y compris des sites dominés par le Bouquetin comme Belvis (960m) ou bien évidemment La Vache $(578 \mathrm{~m})$. L'implantation de la plupart des gisements à proximité de vallées peu encaissées permettant de profiter à la fois des espèces vivant dans les plaines et des espèces plus montagneuses n'est probablement pas étrangère à cela.

\section{CONCLUSION}

Malgré une importante dynamique de recherche depuis plusieurs années, le Badegoulien et le Magdalénien inférieur restent à ce jour assez peu documentés dans les Pyrénées. Le nombre de sites augmente avec le Magdalénien moyen mais les occupations semblent essentiellement cantonnées dans la zone de piémont. Au Magdalénien supérieur les sites sont fréquemment localisés à plus de 500m, sans pour autant dépasser les 1000m 
d'altitude. Un regain d'intérêt pour l'Azilien pyrénéen (voir par exemple FAT CHEUNG, et al., 2014) devrait prochainement permettre de mieux cerner cette période.

La disponibilité en ressources animales particulières au milieu montagnard (Caprinés rupicoles, Oiseaux et Poissons), semble bien avoir été l'un des moteurs de la reconquête des Pyrénées après le Dernier Maximum Glaciaire, en particulier à partir du Magdalénien supérieur. De nombreux gisements témoignent par ailleurs d'une exploitation complémentaire des gibiers montagnards et d'espèces de plaine et c'est ainsi la variété des biotopes environnants qui pourrait avoir motivé l'installation des groupes humains. L'acquisition d'autres ressources - lithiques ou végétales, par exemple également pu jouer un rôle important dans l'installation des hommes dans cette région.

Par rapport aux Alpes, la limite altitudinale des occupations pyrénéennes du Pléni- et du Tardiglaciaire semble beaucoup plus faible. Dans les Alpes, les stations les plus élevées correspondent souvent à des haltes de chasse spécialisées dans l'acquisition de marmottes. Ce gibier ne semble en revanche pas avoir été exploité dans les Pyrénées à cette époque. Sa rareté - voire son absence - sur le versant nord des Pyrénées au Tardiglaciaire pourrait expliquer le faible investissement des sommets pyrénéens à la fin du Paléolithique.

\section{REMERCIEMENTS}

Michel Barbaza, Jean-Marc Pétillon, Robert Simonnet. Fondation Fyssen, ANR Magdatis (2011 BSH3 0005), SRA Midi-Pyrénées

\section{BIBLIOGRAPHIE}

BARBAZA, M

1996 Le Magdalénien supérieur final et l'Azilien dans les Pyrénées centrales. La grotte-abri du Moulin à Troubat (Hautes-Pyrénées), en DELPORTE, H. \& CLOTTES, J. Pyrénées préhistoriques, Actes du 118e congrès National des sociétés historiques et scientifiques, PAU 1993, Paris. 311-326. CTHS.

2011 Environmental Changes And Cultural Dynamics On The Northen Slope Of The Pyrenees During The Younger Dryas. Quaternary International 242(2), 313-327.

BAUMANN, M

2006 Etude typo-technologique de l'industrie en matières dures animales du site magdalénien d'Aurensan. Mémoire de Master I, Préhistoire - Ethnologie - Anthropologie. Université Paris I. 2 v.

BOUCHARD, L.

2008 L'avifaune du site tardiglaciaire de Rhodes // (Ariège): approche taphonomique, archéozoologique et paléoenvironnementale. Master II. Université de Bordeaux I.
BRIDAULT, A., CHAIX, L., PION, G., OBERLIN, C., THIÉBAULT, S. \& ARGANT, J.

2000 Position chronologique du Renne (Rangifer tarandus L.) à la fin du Tardiglaciaire dans les Alpes du Nord françaises et le Jura méridional, en Le Paléolithique supérieur récent: nouvelles données sur le peuplement et l'environnement. Mémoire Société Préhistorique française XXVIII, 47-57.

\section{BRIDAULT, A. \& CHAIX, L.}

2009 Réflexions sur la recomposition des spectres fauniques dans le massif jurassien et les Alpes françaises du nord durant le Tardiglaciaire, en PION, G. \& MEVEL, L.. La fin du Paléolithique supérieur dans les Alpes du nord françaises et le Jura méridional. Approches culturelles et environnementales. 59-72. Mémoire de la Société Préhistorique française.

BRUGAL, J.-P., BRIDAULT, A., GUADELLI, J.-L. \& VIGNE, J.-D. 2004 Distribution des grands mammifères en France aux deux derniers extrêmes climatiques (18 Ka et $8 \mathrm{Ka}$ ) en Actes du XIVe Congrès UISPP, Liège, Sessions générales et posters, section 3. 39-48. BAR International Series 1271.

CLOT, A.

1982 Paléontologie des mammifères, en OMNÈS, J. La grotte ornée de Labastide (Hautes-Pyrénées). 206-255.

1983 Déterminations de paléontologie quaternaire dans le bassin de l'Adour (Première série). Cahiers du Groupe Archéologique des Pyrénées Occidentales 3, 121-139.

1984 Faune de la grotte préhistorique du bois du Cantet (Espèche, Hautes-Pyrénées, France). Munibe Antropología y Arqueología 36, 33-50.

\section{CLOT, A. \& DURANTHON, F.}

1990 Les mammifères fossiles du quaternaire dans les Pyrénées. Publications du Muséum d'Histoire Naturelle de Toulouse.

CLOT, A. \& OMNÈS, J.

1979 Premiers datages radiocarbone du Magdalénien des Hautes-Pyrénées. Bulletin de la Société Préhistorique Française 76, 324-339.

\section{COSTAMAGNO, S.}

2005 Mobilité, territoires de chasse et ressources animales au Magdalénien final en contexte pyrénéen: le niveau $7 a$ de la grotte-abri du Moulin (Troubat, Hautes-Pyrénées), en JAUBERT, J. \& BARBAZA, M. Territoires, déplacements, mobilité, échanges. Actes du 126eme congrés national des sociétés historiques et scientifiques, Toulouse, du 9 au 14 avril 2001, Paris. 371-383. C.T.H.S.

COSTAMAGNO, S., COCHARD, D., FERRIÉ, J.-G., LAROULANDIE, V., CAZALS, N., LANGLAIS, M., VALDEYRON, N., DACHARY, M., BARBAZA, M., GALOP, D., MARTIN, H. \& PHILIBERT, S.

2008 Nouveaux milieux, nouveaux gibiers, nouveaux chasseurs? Évolution des pratiques cynégétiques dans les Pyrénées du Tardiglaciaire au début du Postglaciaire. Bulletin de la Société Préhistorique Française 105(1), 17-27. 


\section{COSTAMAGNO, S. \& LAROULANDIE, V.}

2004 L'exploitation des petits vertébrés dans les Pyrénées françaises du Paléolithique au Mésolithique: un inventaire taphonomique et archéozoologique, en BRUGAL, J.-P. \& DESSE, J. Petits animaux et sociétés humaines. Du complément alimentaire aux ressources utilitaires. XXIVe rencontres internationales d'archéologie et d'histoire d'Antibes. 403-416. Éditions APDCA. Antibes.

DELMAS, M., CALVET, M., GUNNELL, Y., BRAUCHER, R. \& BOURLĖS, D.

2011 Palaeogeography and 10Be exposure-age chronology of Middle and Late Pleistocene glacier systems in the northern Pyrenees: Implications for reconstructing regional palaeoclimates. Palaeogeography, Palaeoclimatology, Palaeoecology 305(1-4), 109-122.

\section{DELPECH, F.}

1999 Biomasse d'Ongulés au Paléolithique et inférences sur la démographie. Paleo 11, 19-42.

\section{DELPECH, F. \& LE GALL, O.}

1983 La faune magdalénienne de la grotte des Eglises, Ussat, Ariège. Bulletin de la Société préhistorique de l'Ariège 38, 91-118.

FAT CHEUNG, C., CHEVALLIER, A., BONNET-JACQUEMENT, P., LANGLAIS, M., FERRIÉ, J.-G., COSTAMAGNO, S., KUNTZ, D., LAROULANDIE, V., MALLYE, J.-B., VALDEYRON, N. \& BALLISTA, S.

2014 Comparaison des séquences aziliennes entre Dordogne et Pyrénées. État des travaux en cours, en LANGLAIS, M., NAUDINOT, N. \& PERESANI, M. Les groupes culturels de la transition Pléistocène-Holocène entre Atlantique et Adriatique. Actes de la séance de la Société préhistorique française de Bordeaux, 24-25 mai 2012, Paris. 17-44. Société Préhistorique Française.

FONTANA, L.

1999 Mobilité et subsistance au Magdalénien dans le Bassin de l'Aude. Bulletin de la Société préhistorique française 96(2), 175-190.

2003 Characterization and exploitation of the Arctic Hare (Lepus timidus) during the Magdalenian: surprising data from Gazel Cave (Aude, France)?, en COSTAMAGNO, S. \& LAROULANDIE, V. Mode de vie au Magdalénien: Apports de l'archéozoologie. Actes du XIVè Congrès UISPP de Liège (2001). 101-118. BAR International Series 1144 .

\section{FOSSE, P}

1992 La grande faune d’Enlène EDG. Remarques paléontologiques et archéozoologiques. Bulletin de la Société préhistorique de l'Ariège 47, 195-220.

\section{GARDEISEN, A.}

2007 La faune de mammifères des niveaux aziliens de la Balma de la Margineda, en GUILAINE, J. \& MARTZLUFF, M. Les Excavaciones a la Balma de la Margineda (1979-1991), Servei de Recerca Historica, Patrimoni Cultural d'Andorra, Min. d'Afers socials i cultura. 492522. v. IV. Govern d'Andorra, Institut d'Estudis Andorrans, Centre de Perpinya.
HARRISON, S. P. \& SANCHEZ GOÑI, M. F.

2010 Global patterns of vegetation response to millennial-scale variability and rapid climate change during the last glacial period. Quaternary Science Reviews 29(21-22), 2957-2980.

\section{JALUT, G. \& TURU I MICHELS, V.}

2009 La végétation des Pyrénées françaises lors du dernier épisode glaciaire et durant la transition glaciaire-interglaciaire (Last Termination), en FULLOLA, J. M., VALDEYRON, N. \& LANGLAIS, M. Els pirineus i les àrees circumdants durant el tardiglacial. Mutacions i filiacions tecnoculturals, evolució paleoambiental (16000-10000 $B P)$. XIV Colloqui internacional d'arqueologia de Puigcerdà. 129-149. Homenatge al professor Georges Laplace. Institut d'estudis Ceretans. Puigcerdà.

LANGLAIS, M., PÉTILLON, J.-M., BEAUNE (de), S., CHAUVIËRE, F.-X., LETOURNEUX, C., SZMIDT, C., BEUKENS, R. \& DAVID, F.

2010 Une occupation de la fin du dernier maximum glaciaire dans les Pyrénées: le Magdalénien inférieur de la grotte des Scilles (Lespugue, Haute-Garonne). Bulletin de la Société Préhistorique Française 107(1), 5-51.

\section{LAROULANDIE, V.}

1998 Etude archéozoologique et taphonomique des Lagopèdes des saules de la grotte des Eglises (Ariège). Anthropozoologica 28, 45-54.

2000 Taphonomie et Archéozoologie des Oiseaux en Grotte: Applications aux Sites Paléolithiques du Bois-Ragot (Vienne), de Combe Saunière (Dordogne) et de La Vache (Ariège). Thèse de l'Université de Bordeaux-l.

2003 Exploitation des oiseaux au Magdalénien en France: état des lieux, en COSTAMAGNO, S. \& LAROULANDIE, $\checkmark$. Mode de vie au Magdalénien: les apports de l'archéozoologie, actes du XIVe congrès de UISPP, septembre 2001, Liège. 129-138. British Archaeological Reports International Series 1144. Oxford.

2005 Bird Exploitation Pattern: the Case of Ptarmigan Lagopus sp. in the Upper Magdalenian Site of La Vache (Ariège, France), en GRUPE, G. \& PETERS, J. Eathers, grit and symbolism. Birds and humans in the ancient Old and New Worlds. Proceedings of the 5th Meeting of the ICAZ Bird Working Group, Munich, 26-28 July 2004. 165-178. Verlag Marie Leidorf. Rahden/Westf. Documenta Archaeobiologiae 3.

2009 De la plume à l'œuf: exploitation des ressources aviaires au Magdalénien dans le Sud de la France, en FONTANA, L., CHAUVIERE F.-X. \& Bridault A., in In search of Total Animal Exploitation. Cases Studies in Upper Palaeolithic and Mesolithic. Proceedings of the XVth UISPP World Congress, Lisbon, 4-9 September 2006, Session C 61, v. 42. 71-89. J. \& E. Hedges. Oxford. BAR International Series 2040.

LE GALL, O.

1999 Ichtyologie et pêches préhistoriques. Quelques données de l'Europe occidentale. Thèse d'État. Université de Bordeaux-I.

2003 Des Magdaléniens et... des poissons, XIV Congrès UISPP, Liège, 2-8 septembre 2001, en COSTAMAGNO, S. \& LAROULANDIE, V. Mode de vie au Magdalénien: les apports de l'archéozoologie. 119-128. Oxford. BAR International Series 1144

2007 Els peixos de la Balma de la Margineda (Capes de la 7 a la 10), en GUILAINE, J., BARBAZA, M. \& MARTZLUFF, M. Les excavacions a la Balma de la Margineda (1979-1991) v. 4. 532-539. Principat d'Andorra, Ministeri d'Afers Social Cultura Govern d'Andorra. 
LEQUATRE, P.

1994 Le monde animal. La faune des grands vertébrés, in BINTZ, P. (dir.). Les grottes Jean-Pierre 1 et 2 à SaintThibaud-de-Couz (Savoie). Première Partie. 197-204. Gallia Préhistoire 36.

MARTIN, H.

1994 Nouveaux milieux, nouveaux chasseurs: une approche des comportements au post-glaciaire à travers l'étude des saisons de capture du gibier. Thèse de Doctorat de I'Université de Toulouse II - Le Mirail.

2007 Recherche de la saison de capture des bouquetins par les Aziliens de la Balma Margineda, en GUILAINE, J. \& MARTZLUFF, M. Les Excavaciones a la Balma de la Margineda (1979-1991) v. IV. 524-539. Servei de Recerca Historica, Patrimoni Cultural d'Andorra, Min. d'Afers socials i cultura / Govern d'Andorra, Institut d'Estudis Andorrans, Centre de Perpinya.

MARTIN, H. \& MARTIN, B.

2010 Note concernant les observations relatives aux études de saisonnalités réalisées sur les restes dentaires des couches C7 et C7bis de la grotte Gazel (Sallèles-Cabardès, Aude). Préhistoire du Sud-Ouest 18(2), 153-160.

MEVEL, L.

2010 Des sociétés en mouvement: nouvelles données sur l'évolution des comportements techno-économiques des sociétés magdaléniennes et aziliennes des Alpes du nord françaises (14000 - 11000 BP). Thèse de Doctorat. Université Paris Ouest Nanterre La Défense.

MOIGNE, A.M.

2003 Les faunes de grands mammifères de la grotte des Conques à Vingrau, en BAILS, H. Les Conques: des chasseurs magdaléniens et leur territoire, Liège. 75103. ERAUL.

MONIN, G., GRICCO, C. \& TOMÉ, C.

2006 Stratégie d'exploitation d'un écosystème alpin au Tardiglaciaire. Les chasseurs de marmottes du Vercors, en MIRAS, Y. \& SURMELY, F. Environnement et peuplement de la moyenne montagne du Tardiglaciaire à nos jours. Actes de la table ronde internationale de Pierrefort (Cantal) du 19 au 20 juin 2003. 29-50.

NAUGHTON, F., SÁNCHEZ GOÑI, M. F., KAGEYAMA, M. BARD, E., DUPRAT, J., CORTIJO, E., DESPRAT, S., MALAIZÉ, B., JOLY, C., ROSTEK, F. \& TURON, J. L.

2009 Wet to dry climatic trend in north-western Iberia within Heinrich events. Earth and Planetary Science Letters 284(3-4), 329-342.

ORLIAC, M.

1975 La grotte de Tourasse-Saint-Martory (Haute-Garonne). Bulletin de l'Association française pour l'étude du quaternaire 12(3-4), 189-190.

PAILHAUGUE, N.

1996 Faune et saisons de chasse de la salle Monique, grotte de la Vache (Alliat, Ariège), en DELPORTE, H. \& CLOTTES, J. Pyrénées préhistoriques, arts et sociétés, Actes du 118 e Congrès national des sociétés historiques et scientifiques (Pau, 1993). 173-192. C.T.H.S. Paris.
2004 La faune de la Salle Monique, en CLOTTES, J., DELPORTE, H. \& BUISSON, D. La grotte de la Vache (Ariège). I. Les occupations du Magdalénien. Réunion des Musées Nationaux. 73-139. C.T.H.S. Paris.

PATOU, M.

1984 La faune de la galerie Rive Droite du Mas d'Azil (Ariège): données paléoclimatiques et paléthnographiques. Bulletin de la Société Préhistorique Française 81(1012), 311-319.

PÉTILLON, J.M., LANGLAIS, M., BEAUNE (DE), S., CHAUVIĖRE, F.-X., LETOURNEUX, C., SZMIDT, C., BEUKENS, R. \& DAVID, F.

2008 Le Magdalénien de la grotte des Scilles (Lespugue, Haute-Garonne). Premiers résultats de l'étude pluridisciplinaire de la collection Saint-Périer. Antiquités nationales 39, 57-71.

PÉTILLON, J.-M., LANGLAIS, M., KUNTZ, D., NORMAND, C., BARSHAY-SZMIDT, C., COSTAMAGNO, S., DELMAS, M., LAROULANDIE, V. \& MARSAN, G.

2015 The human occupation of the northwestern Pyrenees in the Late Glacial: New data from the Arudy basin, lower Ossau valley. Quaternary International 364(0), 126-143.

PHOCA-COSMETATOU, N.

2005 Landscape use in Northeast Italy during the Upper Palaeolithic. Preistoria Alpina 41, 23-49.

2009 Specialisation \& diversification: a tale of two subsistence strategies from Late Glacial Italy. Before farming 2009-3 article 2

PIETTE, E.

1895 Hiatus et lacune. Vestiges de la période de transition dans la grotte du Mas-d'Azil. Bulletins de la Société d'anthropologie de Paris IV ${ }^{\circ}$ Série 6, 235-267.

RASMUSSEN, S. O., ANDERSEN, K. K., SVENSSON, A M., STEFFENSEN, J. P., VINTHER, B., CLAUSEN, H. B., SIGAARD-ANDERSEN, M. L., JOHNSEN, S. J., LARSEN, L. B., DAHL-JENSEN, D., BIGLER, M., RÖTHLISBERGER, R. R., FISCHER, H., GOTO-AZUMA, K., HANSSON, M. E. \& RUTH, U.

2006 A new Greenland ice core chronology for the last glacial termination. Journal of Geophysical Research 111D6, 1984-2012.

RASMUSSEN, S. O., BIGLER, M., BLOCKLEY, S. P., BLUNIER, T., BUCHARDT, S. L., CLAUSEN, H. B., CVIJANOVIC, I., DAHL-JENSEN, D., JOHNSEN, S. J., FISCHER, H., GKINIS, V., GUILLEVIC, M., HOEK, W. Z., LOWE, J. J., PEDRO, J. B., POPP, T., SEIERSTAD, I. K., STEFFENSEN, J. P., SVENSSON, A. M., VALLELONGA, P., VINTHER, B. M., WALKER, M. J. C., WHEATLEY, J. J. \& WINSTRUP, M.

2014 A stratigraphic framework for abrupt climatic changes during the Last Glacial period based on three synchronized Greenland ice-core records: refining and extending the INTIMATE event stratigraphy. Quaternary Science Reviews 106(0), 14-28.

SOAVE, R.

1998 Les chasseurs du Taï 2: Etude archéozoologique d'un site Epipaléolithique de la Drôme. Mémoire de maîtrise, Université Lumière - Lyon 2. 
SZMIDT, C., LAROULANDIE, V., DACHARY, M., LANGLAIS, M. \& COSTAMAGNO, $S$.

2009 Harfang, Renne et Cerf: nouvelles dates 14C par SMA du Magdalénien supérieur du Bassin aquitain au Morin (Gironde) et Bourrouilla (Pyrénées-Atlantiques). Bulletin de la Société Préhistorique Française 106(3), 583-587.

TOMÉ, C.

2005 Les Marmottes de la grotte Colomb (Vercors - France). Revue de Paléobiologie 10 vol. spécial, 11-21.

TOMÉ, C. \& CHAIX, L.

2003 La chasse et l'exploitation des marmottes dans les Alpes occidentales et le Jura du sud de la fin du Pléistocène à l'Holocène, en RAMOUSSE, R., ALLAINE, D. \& LE BERRE, M. Stratégies adaptatives et diversité chez les marmottes, International network on Marmots. 77-84.

\section{VILETTE P.}

1999 Bilan provisoire sur la chasse aux oiseaux pendant le leptolithique dans le sud de la France, en Sacchi D. (Dir.). Les faciès leptolithiques du nord-ouest méditerranéen: milieux naturels et culturels. 267-276. Actes du XXIVe Congrès Préhistorique de France, 26-30 septembre 1994, Carcassonne. Société Préhistorique Française.

WALKER, M. J. C.

1995 Climatic changes in Europe during the last Glacial/Interglacial transition. Quaternary International 28, 63-76. 